\title{
Effectuez le check-up de votre LPP !
}

Malgré le fait que la majeure partie de la fortune privée du corps médical se trouve dans une caisse de pension, ce domaine est souvent négligé. Grâce à une vérification régulière, la solution choisie sera adaptée à la stratégie de placement et de prévoyance personnelle. Un objectif supplémentaire est l'optimisation de la situation fiscale. Pour ce faire, profitez de nos prestations «check-up LPP» et laissez-vous conseiller par un partenaire de confiance FMH Services (Insurance).

\section{CHECK-UP LPP} \section{T FMHSERVICES}

$\square \quad$ Je désire me faire conseiller sans engagement. Veuillez prendre contact avec moi afin de déterminer un entretien.

$\square \quad$ Veuillez m'envoyer sans frais une offre de comparaison. (Merci de joindre une copie de votre certificat de prévoyance.)

$\square \quad$ Veuillez m'appeler pour un conseil personnalisé.

Prénom / nom

Adresse

NPA / lieu

Téléphone privé / cabinet

Atteignable le plus facilement

Adresse e-mail

Published under the copyright license "Attribution - Non-Commercial - NoDerivatives 4.0". No commercial reuse without permission.

\section{INSURANCE}

\section{Roth Gygax \& Partner AG}

Moosstrasse 2, 3073 Gümligen

Tél. 0319595000 - Fax 0319595010

mail@fmhinsurance.ch - www.fmhinsurance.ch

Roth Gygax \& Partner AG est une entreprise de conseil indépendante sur le plan juridique et économique recommandée par la société coopérative FMH Services.

See: http://emh.ch/en/services/permissions.html 